University of Wollongong

Research Online

Australian Institute for Innovative Materials -

Papers

Australian Institute for Innovative Materials

2013

Scalable one-step wet-spinning of graphene fibers and yarns from liquid crystalline dispersions of graphene oxide: towards multifunctional textiles

Rouhollah Jalili

University of Wollongong, rjalili@uow.edu.au

Seyed Hamed Aboutalebi

University of Wollongong, sha942@uowmail.edu.au

Dorna Esrafilzadeh

University of Wollongong, dornae@uow.edu.au

Roderick L. Shepherd

University of Wollongong, rods@uow.edu.au

Jun Chen

University of Wollongong, junc@uow.edu.au

See next page for additional authors

Follow this and additional works at: https://ro.uow.edu.au/aiimpapers

Part of the Engineering Commons, and the Physical Sciences and Mathematics Commons

Research Online is the open access institutional repository for the University of Wollongong. For further information contact the UOW Library: research-pubs@uow.edu.au 


\title{
Scalable one-step wet-spinning of graphene fibers and yarns from liquid crystalline dispersions of graphene oxide: towards multifunctional textiles
}

\begin{abstract}
Key points in the formation of liquid crystalline (LC) dispersions of graphene oxide (GO) and their processability via wet-spinning to produce long lengths of micrometer-dimensional fibers and yarns are addressed. Based on rheological and polarized optical microscopy investigations, a rational relation between GO sheet size and polydispersity, concentration, liquid crystallinity, and spinnability is proposed, leading to an understanding of lyotropic LC behavior and fiber spinnability. The knowledge gained from the straightforward formulation of LC GO "inks" in a range of processable concentrations enables the spinning of continuous conducting, strong, and robust fibers at concentrations as low as $0.075 \mathrm{wt} \%$, eliminating the need for relatively concentrated spinning dope dispersions. The dilute LC GO dispersion is proven to be suitable for fiber spinning using a number of coagulation strategies, including non-solvent precipitation, dispersion destabilization, ionic cross-linking, and polyelectrolyte complexation. One-step continuous spinning of graphene fibers and yarns is introduced for the first time by in situ spinning of LC $\mathrm{GO}$ in basic coagulation baths (i.e., $\mathrm{NaOH}$ or $\mathrm{KOH}$ ), eliminating the need for post-treatment processes. The thermal conductivity of these graphene fibers is found to be much higher than polycrystalline graphite and other types of 3D carbon based materials. New insights are provided into the processing of liquid crystalline graphene oxide (GO) dispersion (containing large $\mathrm{GO}$ sheets) demonstrating a facile and scalable production of $\mathrm{GO}$ and reduced $\mathrm{GO}$ fibers and yarns with exciting properties such as high thermal conductivity. These results provide a universal platform for the development of solution-based processing methods, properties, and applications of liquid crystalline GO-based architectures.
\end{abstract}

\section{Keywords}

liquid, step, crystalline, dispersions, oxide, towards, multifunctional, textiles, scalable, wet, one, yarns, spinning, graphene, fibers

\section{Disciplines}

Engineering | Physical Sciences and Mathematics

\section{Publication Details}

Jalili, R., Aboutalebi, S., Esrafilzadeh, D., Shepherd, R. L., Chen, J., Aminorroaya-Yamini, S., Konstantinov, K., Minett, A. I., Razal, J. M. \& Wallace, G. G. (2013). Scalable one-step wet-spinning of graphene fibers and yarns from liquid crystalline dispersions of graphene oxide: towards multifunctional textiles. Advanced Functional Materials, 23 (43), 5345-5354.

\section{Authors}

Rouhollah Jalili, Seyed Hamed Aboutalebi, Dorna Esrafilzadeh, Roderick L. Shepherd, Jun Chen, Sima Aminorroaya-Yamini, Konstantin Konstantinov, Andrew I. Minett, Joselito M. Razal, and Gordon G. Wallace 
DOI: 10.1002/adfm.201300765

Scalable One-Step Wet-spinning of Graphene Fibers and Yarns from Liquid Crystalline Dispersions of Graphene Oxide: Towards Multifunctional Textiles**

By Rouhollah Jalili, Seyed Hamed Aboutalebi, Dorna Esrafilzadeh, Roderick L. Shepherd, Jun Chen, Sima Aminorroaya-Yamini, Konstantin Konstantinov, Andrew I. Minett, Joselito M. Razal* and Gordon G. Wallace*

[*] Dr. R. Jalili, Ms. D. Esrafilzadeh, Dr. R. L. Shepherd, Dr. J. Chen, Dr. J. M. Razal, Prof. G. G. Wallace Intelligent Polymer Research Institute ARC Centre of Excellence for Electromaterials Science AIIM Facility, Innovation Campus University of Wollongong North Wollongong, NSW 2522 (Australia)

E-mail: jrazal@uow.edu.au,gwallace@uow.edu.au

Mr. S. H. Aboutalebi, Dr. S. Aminorroaya-Yamini, Dr. K. Konstantinov Institute for Superconducting and Electronic Materials

AIIM Facility, Innovation Campus University of Wollongong

North Wollongong, NSW 2522 (Australia)

A/Prof. A. I. Minett

Laboratory for Sustainable Technology

School of Chemical and Biomolecular Engineering

University of Sydney

Sydney NSW 2006 (Australia)

Keywords: graphene oxide, liquid crystal, fiber, multifunctional textiles, rheology

\begin{abstract}
In the present work, we address key points in the formation of liquid crystalline (LC) dispersions of graphene oxide (GO) and their processability via wet-spinning to produce long lengths of micron dimensional fibers and yarns. Based on rheological and polarized optical microscopy investigations, a rational relation between GO sheet size and polydispersity, concentration, liquid crystallinity and spinnability is proposed leading to the understanding of
\end{abstract}


lyotropic LC behavior and fiber spinnability. The knowledge gained from our straightforward formulation of LC GO “inks” in a range of processable concentrations enabled us to spin continuous conducting, strong and robust fibres at concentrations as low as 0.075 wt. \% eliminating the need for relatively concentrated spinning dope dispersions. This concentration is the lowest ever reported value for spinning of any types of liquid crystals arising from any types of colloidal dispersion. The dilute LC GO dispersion was proven suitable for fiber spinning using a number of coagulation strategies including non-solvent precipitation, dispersion destabilization, ionic cross-linking and polyelectrolyte complexation. Employing exceptionally large GO sheets used in this study (up to $100 \mu \mathrm{m}$ with a mean size of $37 \mu \mathrm{m}$ ) resulted in fibers with tensile strength of $442 \mathrm{MPa}$, Young's modulus of $22.6 \mathrm{GPa}$, toughness of $4.8 \mathrm{~J} \mathrm{~g} \mathrm{~g}^{-1}$ which are considerably higher than those reported for graphene-derived architectures. Furthermore, we introduce one step continuous spinning of graphene fibers and yarns for the first time by in situ spinning of LC GO in basic coagulation baths (i.e. $\mathrm{NaOH}$ or $\mathrm{KOH}$ ) eliminating the need for post-treatment processes. The thermal conductivity of these graphene fibres (1435 $\mathrm{W} \mathrm{m}^{-1} \mathrm{~K}^{-1}$ ) was found to be much higher than polycrystalline graphite and any other types of 3D carbon based materials.

\section{Introduction}

The discovery of graphene has spawned a significant interest in carbon nanomaterial research. $[1,2]$ The extraordinary properties of this intriguing material have been used to provide advantage in numerous areas including composites, energy storage and conversion, nanoelectronics, sensors, biosensors, as a support for catalysts and transparent conducting films. ${ }^{[1,3-9]}$ However, in all of these studies, the performance levels obtained are limited by the ability to translate the properties inherent in graphene nanocomponents into the macroscopic structures needed for practical applications. ${ }^{[4]}$ In this context, the recent discovery of liquid crystalline (LC) behavior of graphene oxide (GO) dispersions in various 
organic, ${ }^{[8]}$ and aqueous media brings added control to the assembly of larger structures using the chemical process approach. ${ }^{[10-16]}$ The LC state can be used to direct the ordered assembly of nanocomponents in macroscopic structures via simple methods like wet-spinning. ${ }^{[14,17]}$ The orientation induced by the anisotropic phase combined with the additional contribution of the flow field in fiber spinning methods provide a viable approach to the creation of highly ordered domains and results in strong fibers without the need for post-spinning drawing treatments. ${ }^{[18,19]}$ This advantage has been elegantly exploited in nature (e.g. spiders) where relatively benign spinning conditions and minimal forces are used to 'freeze' spider silk protein orientation and achieve outstanding mechanical properties. ${ }^{[19]}$ LC carbon nanotube (CNT) solutions in superacids have been used to spin high performance synthetic fibers, ${ }^{[18]}$ with the order from the LC phase in the dispersion retained in the fiber. Recent reports described fiber wet-spinning of aqueous LC GO dispersions. ${ }^{[14,17]}$ However, the concept of one-step large-scale fabrication of graphene fibers which is crucial to realizing multifunctional textiles required for industry-based applications has yet to be demonstrated.

Here, we developed a one-step large scale fabrication method to produce graphene fibers via a facile continuoes in-situ reduction in basic coagulation baths (i.e. $\mathrm{NaOH}$ or $\mathrm{KOH}$ ). We examine the relationship between the size of GO sheets, the concentration of the dispersion and liquid crsytallinity. We also develop further understanding in the required criteria to correlate processability with LC behavior, aspect ratio and the dispersion concentration to provide a viable platform for spinning of LC GO. We demonstrate a striking result that highlits the importance of GO sheet size and polydispersity in generating wet-spinnable LC GO dispersions from very low spinning dope concentrations (as low as 0.075 wt. \%). The new knowledge gained through rheological investigations provides a sound explanation as to why continuous spinning of binder-free GO fibers is enabled by the LC behavior at this very low concentration. 


\section{Results and discussions}

\subsection{Birefringence, rheological behavior and spinnability of GO dispersions}

Based on our previously described GO synthesis protocols and completely eliminating the sonication previously deemed necessary in the complete exfoliation of GO sheets, ${ }^{[10]}$ we have produced ultra-large GO sheets with lateral size of up to $100 \mu \mathrm{m}$ (Figure. 1). A SEM survey of 250 GO sheets confirmed that the majority of the lateral GO sheet sizes are in the tens of micrometers domain with an average lateral sheet size of $37 \mu \mathrm{m}$ (Figure. S1). AFM analysis confirmed that these GO sheets are indeed monolayer with an apparent thickness of $\sim 0.80 \mathrm{~nm}$ (Figure. 1a). Also apparent in the AFM images is the highly wrinkled nature of the GO sheets with $\sim 3$ to $12 \mathrm{~nm}$ perturbations. The presence of wrinkled sheets could be explained by the formation of hydrogen bonds among the oxygenated functional groups, ${ }^{[20]}$ that are also believed to facilitate full delamination of monolayer sheets in aqueous dispersions. ${ }^{[9]}$ The polydispersity $(\sigma)$ of the system was determined by dividing the standard deviation of the diameter distribution $(23 \mu \mathrm{m})$ by the mean $(37 \mu \mathrm{m})$. In a polydisperse GO dispersion, large GO sheets generate excluded volume for small sheets giving rise to entropic rearrangement to form long range ordering resembling a liquid crystalline state. The critical theoretical volume fraction $(\Phi)$ for the transition between isotropic to nematic phase can be calculated based on Equation 1 which served as a model system for liquid crystal phases of charged colloidal platelets, ${ }^{[21,22]}$

$$
\Phi=\frac{3}{8} \sqrt{3} \frac{L}{D} \frac{1+\sigma^{2}}{1+3 \sigma^{2}} \rho D^{3}
$$

The dimensionless number density $\left(\rho \mathrm{D}^{3}\right)$ was experimentally found to be $\rho_{\text {iso }} \mathrm{D}^{3}=2.7$ and $\rho_{\text {nem }} \mathrm{D}^{3}=4.3$ for isotropic and nematic transition concentrations, respectively. However, there are some assumptions in this model which should be taken into account for any further improvement of the theoretical foundation. These assumptions state that the particles are rigid and the diameter distribution is symmetric. The sheet thickness (L) was estimated to be 0.81 
$\mathrm{nm}$ based on AFM measurements and GO sheet density was considered to be $2.2 \mathrm{~g} \mathrm{~cm}^{-3}$ based on the literature. ${ }^{[10]}$ The above equation gave a theoretical biphasic region between 0.05 to $0.09 \mathrm{mg} \mathrm{ml}^{-1}$. Aqueous GO dispersions at concentrations ranging from 0.10 to $5.00 \mathrm{mg} \mathrm{ml}^{-1}$ were examined using polarized optical microscopy and displayed that nematic phase start to form at GO concentrations close to $0.25 \mathrm{mg} \mathrm{ml}^{-1}$ (Figure. 2), which is higher than theoretical calculations $\left(0.09 \mathrm{mg} \mathrm{ml}^{-1}\right)$. This difference mainly arises as a result of flexibility of GO sheets, which results in the wrinkling behavior. The wrinkling can also have profound effect on the charge concentrations on the surface of GO sheets leading to electrostatic anomaly on the surface and localization of electrostatic charges on some preferred regions. Also the asymmetry of size distribution and shape irregularity can play important roles in the deviation of theoretical estimations from experimental values.

Wet-spinning experiment (Figure. S3) was involved injecting LC GO dispersion into a coagulation bath to produce the gel-state GO fiber. Below $0.25 \mathrm{mg} \mathrm{ml}^{-1}$, GO dispersions were completely isotropic and un-spinnable (i.e. GO sheets simply spread into the coagulation bath). At higher concentration (between 0.25 to $0.75 \mathrm{mg} \mathrm{ml}^{-1}$ ), transition of biphasic to fully nematic phase is observed (i.e. when isotropic and nematic phases co-exist). This biphasic concentration range only demonstrated partial spinnability in fiber spinning processes. This partial spinnability is characterized by the weak cohesion of the GO dispersion upon injecting in the coagulation bath, which resulted in short lengths of gel-state GO fibers. Also observed in this concentration range is that the nematic phase volume fraction $\left(\varphi_{\text {nem }}\right)$ increased with GO concentration. Using GO concentration of $\geq 0.75 \mathrm{mg} \mathrm{ml}^{-1}$, when fully nematic phase forms, in fiber wet-spinning resulted in long lengths of robust gel-state fibers. GO concentrations of $0.75 \mathrm{mg} \mathrm{ml}^{-1}$ to $5 \mathrm{mg} \mathrm{ml}^{-1}$ showed similar ease of spinnability.

Rheological investigations were carried out to correlate LC behavior, GO concentration and spinnability. The rheological behavior of the LC GO dispersions exhibited that of non- 
Newtonian liquids with flow curves exhibiting yield values (the lowest shear stress necessary to produce viscous flow) ${ }^{[23]}$ similar to the rheological behavior of plastic fluids. ${ }^{[24]}$ To better illustrate this behavior, the flow curves of $5 \mathrm{mg} \mathrm{ml}^{-1}$ GO dispersion are shown in Figure. 3a. When the flow measurements are repeated, the yield value on the ascending curve corresponds to that on the first descending curve. This suggests that an orientation of the anisotropic phases took place in the shear field. The lower yield value obtained when the measurements were repeated corresponds to that of the aligned sample. Further confirmation of this plastic behavior is evident from the apparent viscosity $(\eta)$ that approaches $\infty$ as shear rate $(\dot{\gamma})$ approaches 0 , which was found to experimentally scale as $\eta \sim(\dot{\gamma})^{-0.952}$ (Figure. 3-b). It should be noted that the theoretical exponent for plastic materials has a value of -1 . This theoretical value suggests limited structural modifications, such as an alignment of the lamellar planes, but no viscous components. A viscous character would show an exponent value between -1 and 0 . Therefore the slight deviation from the theoretical value of $-1,{ }^{[24]}$ represents the viscous component of the material. The most feasible physical mechanism that can be taken into account for the observed plastic behavior of the LC phase is the sliding of lamellae along the layer of water molecules. Based on thermodynamics, there is no driving force for the relaxation of the structure after sliding along any possible direction tangent to the lamellae. ${ }^{[24]}$ It is also hypothesized that sliding can very likely occur between the water phase lamellae. Therefore, it is safe to assume that at the very first stage (low strain), water is confined between the layers. After the initial confinement of water, the shear stress decreases and then increases with increasing shear rate. This water confinement gives rise to the nonNewtonian behavior observed here. However, upon decreasing the concentration to the region where a mixture of phases can be observed, the exponent value completely deviates from the theoretical value of -1 , demonstrating the loss of plastic component (Figure. 3-b, inset). It is pertinent to mention that at GO concentrations below $0.75 \mathrm{mg} \mathrm{ml}^{-1}$, a sharp deviation in the 
ideal scaling is evident as GO concentration decreased, suggesting the loss of the plastic component and the viscous component becoming more dominant. The prevailing behavior coincided with the emergence of isotropic phase as GO concentration decreased.

Figure. 3-c and 3-d show the yield value of GO dispersion as a function of concentration and nematic phase volume fractions $\left(\varphi_{\text {nem }}\right)$ for the first and second ascending shear stress. Investigations of the rheological behavior of aqueous GO dispersions (also evaluated as fiber spinning solutions) reveal that yield values increase systematically with GO concentration. At concentrations $\leq 0.25 \mathrm{mg} \mathrm{ml}^{-1}$ where $\varphi_{\text {nem }}$ is negligible, there was no difference between the two yield values for the first and second ascending flows. This concentration range (I) was un-spinnable. At $0.25<\mathrm{GO} \leq 0.75 \mathrm{mg} \mathrm{ml}^{-1}$ (II), the difference in yield values observed then increased with $\varphi_{\text {nem }}$ which is in complete agreement with the transition concentration to a fully nematic phase observed in POM studies. Above $0.75 \mathrm{mg} \mathrm{ml}^{-1}$ (III, fully nematic), the flow curves reveal significant difference between yield values that increase almost linearly with GO concentration. In this region, cohesion of the spinning solution dramatically improved and long lengths of robust gel fiber were produced and subsequently dried without breaking. The shear-induced alignment of the LC phase could explain the observed excellent spinnability of the fully nematic LC GO dispersions. The large GO sheet dimensions and aspect ratio (the average lateral size of $\sim 37 \mu \mathrm{m}$ and thickness of $0.8 \mathrm{~nm}$ give a mean aspect ratio of $\sim 45000$ which is much higher than previous reports) ${ }^{[12-14]}$ provide alignment of the sheets within the fibers produced. ${ }^{[15]}$ Of note is that the concentration and viscosity of a spinnable LC GO dispersion can be as low as $0.75 \mathrm{mg} \mathrm{ml}^{-1}$ and $\sim 30 \mathrm{~Pa}$ s, respectively. This concentration was around two orders of magnitude lower than the previously reported value for wet-spinning of LC GO dispersion. ${ }^{[14]}$ Conventionally much higher concentration and viscosity are required for wet-spinning formulations. ${ }^{[25]}$ This low concentration hold promises to a wide range of possibilities including new formulations of LC GO with different materials (CNTs, metallic 
based particles, polymers, etc) that are not accessible in the case of concentrated dispersions because of the viscosity limitations.

In a recent paper, ${ }^{[15]}$ it was demonstrated that giant GO flakes (larger than $10 \mu \mathrm{m}$ ) can undergo a transition to an LC gel-like state. The gel-like LC state showed an unusually defectfree alignment over hundreds of micrometers. In general, a gel is considered as a semi-rigid network in which a liquid is held. ${ }^{[26]}$ However, the gel-like state induced because of ultralarge GO sheets prepared in our study exhibits fundamental characteristics that ordinary gels do not have. These characteristics include exceptional uniformity of the network structure (large domain LC), and the anisotropy arising from having LC in the network, which are the common features of liquid crystalline gel structures (solvent content $>99 \%$ ). ${ }^{[15,26]}$ These unique features enable our as-prepared dispersions to be aligned by shear, as demonstrated by rheological studies, and ensure the preservation of the order even at low concentrations as they form a gel-like LC fiber as a result of wet-spinning.

\subsection{GO and rGO fibers}

LC GO dispersions were found to be versatile for use in various wet-spinning coagulation systems. ${ }^{[25,27-29]} \mathrm{GO}$ dispersions that displayed the LC behavior (i.e. those above $0.75 \mathrm{mg} \mathrm{ml}^{-}$

${ }^{1}$ ) exhibited excellent spinnability in any of the following coagulation methods: non-solvent precipitation, dispersion destabilization using acid, base or salt solutions, ionic cross-linking using divalent cations, and coagulation by amphiphilic or oppositely charged polymers. Representative digital images of fibers produced using the above coagulation strategies are shown in Figure. S4. Note that we identified spinnability to be excellent only when continuous gel-state GO fibers exceeded $10 \mathrm{~m}$ in length. These gel-state GO fibers must also withstand the drying and collection process whereby the gel-state GO fiber is passed through a series of bath containing water, acetone and/or ethanol before being collected dry onto a spool. After drying, reduction of GO to rGO fibers was achieved either by annealing at $220{ }^{\circ} \mathrm{C}$ 
or by chemical treatment with hydrazine vapor. The scalability of this process was demonstrated by using the multi-filament spinning approach to produce GO yarns. This type of fiber spinning was done by replacing 1-hole spinneret with a 50-hole spinneret (with each orifice diameter of $100 \mu \mathrm{m})$. Shown in Figure. 4 is a snapshot of the formation of gel-state GO filaments as they exit the multi-hole spinneret and, at the other end, are being pulled out from the bath for collection.

Polarized optical micrograph of the gel-state GO fibers displayed birefringence (Figure. $5 \mathrm{a}$ ), which suggests highly ordered GO domains are preserved during this initial stage of the spinning process. It is important to note that in the case of GO, the time scale for the formation of mono-domains without applying an external driving force would be infinite. ${ }^{[13]}$ Magnetic field assisted alignment of LC GO is reported to occurr just after 5 hours to form uniform LCs. ${ }^{[13]}$ However, macroscopic alignement was obtained during wet spinning in just a few seconds as a result of gentle shear stress applied through the spinneret due to the presence of giant sheets (mean aspect ratio of 45000 vs 1600) ${ }^{[13]}$ forming large LC domains. LCs with larger domains are known to yield macroscopic materials with higher microstructural order and better properties. ${ }^{[30]}$ In addition, the tension applied through the spinneret oriented the LC domains and rapid coagulation preserved this orientation. In the dry-state fibers, the morphology of the fiber cross-section revealed that GO sheets are stacked in layers with some degree of folding and are orientated in the fiber axis direction (Figure. 5bd).

Analysis of the distinct sharp peak observed at $2 \theta=10.5^{\circ}$ in the XRD pattern of room temperature washed/dried GO fibers suggested that the observed layered GO sheets have a mean $d$-spacing of $\sim 8.42 \AA$ (Figure. 6). This $d$-spacing is similar to GO papers prepared from the same LC GO dispersions (Figure. S1), which confirms that water molecules are retained in the interlamellar GO sheets. XRD analysis of the GO fiber annealed at $220^{\circ} \mathrm{C}$ displayed a 
significantly different XRD pattern but with a $d$-spacing (estimated from the maximum peak to be $\sim 3.71 \AA$ ) close to that of the fully graphitic expanded graphite starting material, which showed a distinct sharp (002) peak at $26^{\circ}$ and a $d$-spacing that corresponds to $\sim 3.32 \AA$. These results suggest that water and the majority of the chemical functionalities in the GO sheets were removed after annealing and/or chemical reduction by hydrazine vapor; indicating the effectiveness of the reduction process. ${ }^{[9,10]}$ The reduction of GO by annealing is further confirmed by the significantly higher electrical conductivity of rGO fiber ( 2844 S m$\left.~^{-1}\right)$ compared to the parent insulating GO fiber.

\subsection{Mechanical properties}

Representative stress-strain curves of the GO fibers spun using various coagulation baths are compared in Figure. 7a and the mechanical properties along with the comparison with previous reports on graphene fibers are summarized in Table 1. It is evident from the results coagulation bath employed and reduction of the GO sheets influence the mechanical properties of GO and rGO fibers.

As-spun GO fibers prepared by ionic cross-linking and polyionic complexation (using the positively charged polymer, chitosan) displayed the highest mechanical properties with Young's modulus (E), ultimate strength $(\delta)$ and toughness $(\mathrm{T})$ of $22.6 \pm 1.9 \mathrm{GPa}, 442 \pm 18 \mathrm{MPa}$ and $4.8 \mathrm{~J} \mathrm{~g}^{-1}$, respectively. In this case, both cross-linking and coagulation by oppositely charged polymers contribute to the enhancement in mechanical properties. However, it should be noted that chitosan is not expected to be able to penetrate into the internal of the fiber and the strengthening, therefore would just happen at the surface. The SEM micrograph presented in Figure. S. 5c clearly shows that the outer layer of the fiber is much more compact than the core of the fiber indicating that the interaction mostly happens at the surface. 
Apart from chitosan, coagulation in $\mathrm{CaCl}_{2}$ which exploits ionic cross-linking using divalent cations $\left(\mathrm{Ca}^{2+}\right)$ also resulted in fibers with extraordinary mechanical properties $(\mathrm{E}=20.1 \pm 2.1$ $\mathrm{GPa}, \delta=412 \pm 30 \mathrm{MPa}$ and $\mathrm{T}=4.8 \mathrm{~J} \mathrm{~g}^{-1}$ ). Divalent metal ions such as $\mathrm{Mg}^{2+}, \mathrm{Ca}^{2+}$ and $\mathrm{Cu}^{2+}$ are known to readily react with carboxylate functional groups typically located at the edges of GO sheets. ${ }^{[31]}$ This chemical interaction can then result in mechanical enhancement of the final architecture. It is expected that these ions can readily diffuse into the core of the fibers resulting in exceptional cross-linking within the fiber.

Partially reduced graphene oxide fibers were fabricated employing continoues one-step wetspinning in alkaline baths. In the case of alkaline baths, although the underlying reduction mechanism is still unclear, it is possible that oxidative debris, which mainly comprises of a mixture of complex aromatic structures containing $\mathrm{COH}$ rich functional groups is stripped from graphene oxide under alkaline conditions. ${ }^{[32,33]}$ Other studies suggest a fast irreversible deoxygenation of graphene oxide under basic conditions. ${ }^{[34]}$ However, irrespective of the mechanisms involved, employing alkaline coagulation baths results in the removal of oxygen multifunctionalities, ${ }^{[32-35]}$ and consequently water from the graphene oxide sheets which in turn results in fiber coagulation and elimination of hydrogen bonds in between GO sheets and weakening of the inter-layer crosslinking. This water elimination leads to slightly lower mechanical properties compared to GO fibers coagulated in acetone bath. For as-prepared GO fibers spun using acetone, the lateral cohesion of the adjacent GO sheets is attributed to the strong van der Waals interactions (from the hydrophobic polyaromatic nanographene domains remaining on the basal planes) ${ }^{[9,20]}$ and the formation of hydrogen bonds (mediated by the oxygenated functional groups and water molecules).

The best as-spun GO fiber strength ( 442 $\mathrm{MPa}$ ) is higher than previously reported GO and rGO -based materials such as GO papers ( 120 MPa), ${ }^{[36]}$ RGOF/PVA fibers ( 120 MPa), ${ }^{\text {[37] }}$ LC GO fibers ( 102 MPa), ${ }^{[14]}$ stretched "ultrastrong“ GO fibers (364 MPa), ${ }^{[17]}$ fibers 
produced from graphene nanoribbons (378 MPa), ${ }^{[38]}$ hydrothermally converted rGO fibers ( $\sim 180 \mathrm{MPa})$ and thermally reduced rGO fibers (420 MPa). ${ }^{[39]}$ In terms of modulus, the GO fiber (22.6 GPa) is inferior to the highest modulus of GO paper (42 GPa) ${ }^{[36]}$ and fibers produced from graphene nanoribbons $(36.2 \mathrm{GPa}),{ }^{[38]}$ but the difference in breaking strain is substantial (3.2 \% for GO fiber vs. $\sim 0.4 \%$ for GO paper and $1.1 \%$ for fibers produced from graphene nanoribbons) resulting in toughness $\left(4.8 \mathrm{~J} \mathrm{~g}^{-1}\right)$ considerably higher than both. ${ }^{[36,38]}$ The strength of as-spun GO fiber is also much higher than single yarns of pure multi-walled CNTs ( 250 MPa). ${ }^{[40]}$ It should be noted that the GO used in this study is much larger

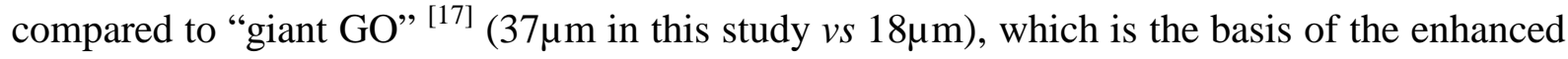
improvement in mechanical properties attained in the current study.

As expected, annealed rGO fibers have lower mechanical properties than the parent GO fibers irrespective of the coagulation bath used. It is pertinent to mention that there are different reduction processes that can be used to reduce graphene oxide. However as a general guideline, irrespective of the mechanisms involved, reducing graphene oxide by either employing annealing at moderate temperatures (upto $220{ }^{\circ} \mathrm{C}$ ) or alkaline coagulation baths results in the removal of oxygen multifunctionalities, ${ }^{[32-35]}$ and consequently water from the graphene oxide sheets which in turn results in the elimination of hydrogen bonds in between GO sheets and weakening of inter-layer crosslinking. ${ }^{[35]}$ This, in turn, results in inferior mechanical properties of reduced graphene oxide compared to parent graphene oxide. However, annealed rGO fibers still displayed flexibility and remained intact even when knots and twists were introduced (Figure. 5 e-f). Notably, reasonable strong electrically conducting rGO fibers were produced in a single step (i.e. no post-spinning annealing treatment is employed). A native conductivity of $\sim 221 \mathrm{~S} \mathrm{~m}^{-1}$ was obtained from $\mathrm{NaOH}$ spun rGO fibers, which displayed modulus and tensile strengths of $~ 11$ GPa and $183 \mathrm{MPa}$, respectively (Table 1). This type of GO reduction process has also been employed in some literature reports as a 
post-treatment process to reduce GO to rGO. ${ }^{[34]}$ In contrast with a previous reports, ${ }^{[14,17]}$ here we show the continuous one step production of rGO fibers using alkaline coagulation baths, which is of technological interest as the need for reduction post-treatment processes can be eliminated. We also investigated the use of hydrazine (by exposing the fiber to hydrazine vapor at $80{ }^{\circ} \mathrm{C}$ ) to reduce the GO fibers. Although this reduction method was effective as evidenced by increase in the electrical conductivity $\left(\sim 250 \mathrm{~S} \mathrm{~m}^{-1}\right)$, the mechanical properties were found to be lower compared to the parent GO fiber. We attributed this decrease in mechanical properties to the significant expansion of GO fiber diameter (up to $400 \%$ ) after hydrazine vapor treatment.

\subsection{Thermal conductivity}

The thermal conductivity of the rGO fiber was determined by a standard four-point probe method in the 50 to $380 \mathrm{~K}$ temperature range (Figure. 7b). Whilst the room temperature thermal conductivity for our rGO fibers $\left(1435 \mathrm{~W} \mathrm{~m}^{-1} \mathrm{~K}^{-1}\right)$ is significantly higher than LC wetspun CNT fibers $\left(20 \mathrm{~W} \mathrm{~m}^{-1} \mathrm{~K}^{-1}\right),{ }^{[18]}$ magnetically aligned CNT films $\left(\sim 220 \mathrm{~W} \mathrm{~m}^{-1} \mathrm{~K}^{-1}\right){ }^{[41]}$ and graphene-based paper $\left(\sim 112 \mathrm{~W} \mathrm{~m}^{-1} \mathrm{~K}^{-1}\right),{ }^{[42]}$ it is however, lower than single layer pristine graphene (up to $5300 \mathrm{~W} \mathrm{~m}^{-1} \mathrm{~K}^{-1}$ ). ${ }^{[43,44]}$ Interestingly, it is also higher than polycrystalline graphite $\left(200 \mathrm{~W} \mathrm{~m}^{-1} \mathrm{~K}^{-1}\right) .{ }^{[43]}$

It is known that the thermal conductivity of multilayer graphene is reduced in comparison to single layer graphene, by an almost linear increment versus the number of layers and an enhancement in the interplanar van der Waals bonding strength. This is considered to be due to interlayer interactions and vibrational restrictions from neighboring layers, which limits the

free vibration of the graphene sheets and imposes resistance on phonon transport. ${ }^{\text {[45] }}$ Therefore, we believe that the thermal transport properties of our rGO fibers are not an indication of the in-plane thermal conductivity of the individual layers due to the tangled nature of the rGO sheets in the fiber and lattice defects that are introduced during GO sheet 
synthesis and subsequent reduction processes. These effects further contribute to the lower thermal conductivity measurements compared to pristine graphene. However, this hypothesis alone does not explain why the thermal conductivity of our rGO is higher than polycrystalline graphite. It should be noted that the measurements were performed along the longitudinal axis of rGo fibers as depicted in Figure. S6. From XRD patterns presented in Figure. 6, it is apparent that the interlayer d-spacing of rGO sheets in the rGO fibers perpendicular to the longitudinal axis $(0.371 \mathrm{~nm})$ is higher than that of raw material $(0.332)$, graphite and CNTs $(0.34 \mathrm{~nm})$, suggesting the presence of remaining multifunctionalities which might act as pillars increasing the interlayer spacing among individual sheets. We suggest that functional groups on the rGO planes enhance interplane bonding strength and consequently limit the vibration of the rGO sheets, which reduces the thermal conductivity compared to graphene; however, this interplane bond strength is less than that of graphite.

\section{Conclusions}

In summary, the new findings enabled us to spin continuous conducting, fully oriented, strong and robust graphene fibers and yarns at practical concentrations as low as 0.075 wt. \% (which is one order of magnitude lower than previously reported and the lowest ever reported value for spinning of any type of colloidal solutions) eliminating the need for relatively concentrated spinning dope dispersions. This low concentration enables a whole range of possibilities including new formulations of LC GO with different materials (CNTs, metallic based particles, polymers, etc) that are not accessible in the case of concentrated dispersions because of the viscosity limitations. The scalability of the process was demonstrated by performing multi-filament spinning and producing fundamentally unlimited length of yarns out of these fibers. The promising properties of GO and rGO fibers presented here are potentially applicable in many specialized technical applications. Fundamental understanding of the GO dispersion chemistry and rheology, the associated fiber spinning processes, and the 
resultant material properties, presented here along with one-step production of rGO fibers will provide scope for the development of next generation advanced GO/rGO-based fiber architectures and associated technologies. The simplicity and the scalability of the processes described here will enable safe and cost-effective large-scale production of GO/rGO fiber to find a place in the smart fiber industry (i.e. fibers that integrate applications in energy, environmental monitoring and biomedical engineering).

\section{Experimental}

GO synthesis: Dry expandable graphite flakes (3772, Asbury Graphite Mills USA) were first thermally treated (at $1050{ }^{\circ} \mathrm{C}$ for 15 seconds) and then used as precursor for GO synthesis following previously described methods [9, 46]. Briefly, expanded graphite (5 g) and sulfuric acid (1 lit) were mixed and stirred in a flask. Then, $\mathrm{KMnO}_{4}(50 \mathrm{~g})$ was added to the mixture dropwise. The mixture was transferred into an ice bath, and Milli-Q (1 lit) water and $\mathrm{H}_{2} \mathrm{O}_{2}$ (250 mL) were poured slowly into the mixture, realizing a colour change of the suspension to light brown. Having stirred for another $30 \mathrm{~min}$, the GO particles were then washed and centrifuged with a $\mathrm{HCl}$ solution (9:1 water: $\mathrm{HCl}$ by volume), then centrifuged again and washed with Milli-Q water until the $\mathrm{pH}$ of the solution became about 5 to 6 . The resultant large GO sheets were dispersed in deionized water by gentle shaking (i.e. without the aid of sonication process).

Characterization of GO sheets: AFM and SEM analysis were carried out by first depositing GO sheets from their dispersions on pre-cleaned and silanized silicon wafer (300 nm $\mathrm{SiO}_{2}$ layer). Silane solution was prepared by mixing 3-Aminopropyltriethoxysilane (Aldrich) with water (1:9 vol) and one drop of hydrochloric acid (Sigma-Aldrich). Pre-cut silicon substrates were silanized by immersing in aqueous silane solution for 30 minutes and then washed thoroughly with Millipore water. GO sheets were deposited onto silanized silicon substrates by immersing a silicon substrate into the GO dispersion (50 $\left.\mu \mathrm{g} \mathrm{ml}^{-1}\right)$ for 5 seconds, then into a 
second container containing Millipore water for 30 seconds and then air-dried. Prior to SEM and AFM analysis, GO sheets were observed under an optical microscope to ensure uniform GO sheet deposition was achieved. As-deposited GO sheets were directly examined by scanning electron microscopy (SEM, JEOL JSM-7500FA). Lateral size distribution of 256 isolated GO sheets were determined from 25 SEM images and analyzed using image analysis software (imageJ, http://rsb.info.nih.gov/ij/). Lateral size of ultra-large GO sheets was defined as the diameter of an equal-area circle. Atomic force microscopy (MFP-3D AFM Asylum Research, CA) was carried out in tapping mode under ambient conditions. Transmission electron microscopy was carried out on GO sheets deposited on holey carbon grid (a droplet from $50 \mu \mathrm{g} \mathrm{ml}^{-1}$ GO suspension) using JEOL JEM-2200FS. X-ray diffraction studies were performed using a powder XRD system (Philips1825) with $\mathrm{CuK} \alpha$ radiation $(\lambda=0.154 \mathrm{~nm})$ operating at $40 \mathrm{keV}$ and with a cathode current of $20 \mathrm{~mA}$.

Characterization of GO dispersions: The birefringence of GO dispersions was examined by polarized optical microscopy (Leica DM EP) operated in transmission mode. The volume fraction of liquid crystalline domains in birefringent solutions was estimated using previously reported methods [22, 47]. Briefly, $75 \mu \mathrm{l}$ of a series of GO dispersion concentration (0.1 to $5.0 \mathrm{mg} \mathrm{ml}^{-1}$ ) was placed in a capillary tube (1.5 mm ID), sealed (using glue) and then left stationary for one week. The volume fraction of the stable birefringent (nematic) phase was then measured by polarized optical microscopy on the seventh day. The rheological properties of aqueous GO dispersions were investigated using a rheometer (AR-G2 TA Instruments) with a conical shaped spindle (angle: $2^{\circ}$, diameter: $40 \mathrm{~mm}$ ). Approximately $600 \mu \mathrm{l}$ of GO dispersions was loaded into the rheometer with great care taken not to shear or stretch the sample. Shear stress and viscosity were measured at shear rates between 0.01 to 10 using logarithmic steps (total 200 points) for two complete (ascending and descending) cycles. Shear rate was kept constant at each point for 2 minutes and data recorded during the last 15 
seconds were reported. Yield value and viscosity (at the lowest shear rate) were obtained and plotted as a function of concentration for the first and second ascending cycles.

Fiber spinning: Two types of fiber wet-spinning systems were utilized as illustrated in Figure. S3. The spinnability of GO dispersions was evaluated using the "petri dish method". In this method, a GO dispersion is injected at flow rates between 5 to $10 \mathrm{ml} \mathrm{h}^{-1}$ into a rotating petri dish (between 30 to $60 \mathrm{rpm}$ ) that contained the coagulation bath. Spinning trials were also carried out using a custom-built wet-spinning apparatus to demonstrate the continuous fiber wet-spinning process. Dried GO fibers were obtained by washing the gel-state GO fibers with water, then 25 vol. \% ethanol or acetone and then air-drying the fiber under tension at room temperature. Reduction of GO fibers (rGO) was carried out by overnight annealing at $220^{\circ} \mathrm{C}$ under vacuum or by exposing the fibers (under tension) to hydrazine vapor at $80^{\circ} \mathrm{C}$ for $3 \mathrm{hrs}$. Characterization of $G O$ and rGO fibers: As the cross-section of the fibers are not perfectly circular, also reported elsewhere [14, 17], we calculated the mechanical properties and electrical conductivity based on the area of a circle with a diameter equal to the longest width (widest diameter) of the irregular fibre (Figure. S5) and denoted the "overestimated area”. However, to have a fair comparison with other literature reports regarding graphene fibers, we have now added calculated mechanical and electrical properties results based on the actual area denoted. The tensile properties of the GO and rGO fibers were measured for 15 samples for each coagulation bath (average and deviation are reported) using a tensile tester (Shimadzu EZ-S) at a strain rate of $0.5 \% \mathrm{~min}^{-1}$. A linear four-point probe conductivity set-up with uniform $2.3 \mathrm{~mm}$ probe spacing was employed to measure the room temperature conductivity of the fibers using a galvanostat current source (Princeton Applied Research Model 363) and a digital multimeter (HP Agilent 34401A). The temperature dependent thermal transport properties were measured in a physical property measurement system (PPMS 6500-Quantum Design), using the standard four-probe method. The pressure of the 
sample chamber was less than $5 \times 10^{-4}$ Torr during the thermal conductivity measurements. Samples were mounted on quartz substrates with known thermal conductivity $\left(1 \mathrm{~W} \mathrm{~m}^{-1} \mathrm{~K}^{-1}\right.$ at room temperature). Therefore, the heat loss to the substrate was neglected. Heat was applied to one end of the fiber by running current through the heater (I+/heat) and heat exits the fiber to the coldfoot. The temperatures $\mathrm{T}_{\text {hot }}$ and $\mathrm{T}_{\text {cold }}$ were measured at the thermometer shoes. These data were employed by the equipment software to measure the thermal conductance (K) of the sample using $\mathrm{K}=\mathrm{kA} / \mathrm{L}$ where $\mathrm{k}$ is thermal conductivity, $\mathrm{A}$ and $\mathrm{L}$ are cross-section of fiber and the distance $(\mathrm{L}=3.13 \mathrm{~mm}$ ) between two thermometer shoes, respectively. It should be noted that the overestimated area was used to determine the thermal conductivity of the samples resulting in an underestimated thermal conductivity values.

\section{Acknowledgements}

The authors thank Tony Romeo for technical assistance, the Australian Research Council (ARC) for financial support, and the ANFF Materials Node for their provision of research facilities. This work was supported by ARC APD Fellowship DP098753 (JMR), ARC Discovery Project DP1093952 (KK and SHA) ARC Discovery Early Career Researcher Award DE130100310 (SAY) and ARC Federation Fellowship (GGW). Expandable graphite was kindly provided by Asbury Carbons. Supporting Information is available online from Wiley InterScience or from the author.

Received: ((will be filled in by the editorial staff))

Revised: ((will be filled in by the editorial staff)) Published online: ((will be filled in by the editorial staff))

[1] Y. Zhu, S. Murali, W. Cai, X. Li, J. W. Suk, J. R. Potts, R. S. Ruoff, Adv. Mater. 2010, 22, 3906.

[2] H. Chang, G. Wang, A. Yang, X. Tao, X. Liu, Y. Shen, Z. Zheng, Adv. Funct. Mater. 2010, 20, 2893.

[3] Y. Zhu, S. Murali, M. D. Stoller, K. J. Ganesh, W. Cai, P. J. Ferreira, A. Pirkle, R. M. Wallace, K. A. Cychosz, M. Thommes, D. Su, E. A. Stach, R. S. Ruoff, Science 2011, 332, 1537. 
[4] H. Chen, M. B. Müller, K. J. Gilmore, G. G. Wallace, D. Li, Adv. Mater. 2008, 20, 3557.

[5] M. G. Mohsen, A. S. Hamed, Y. Nariman, Z. Q. Bin, S. Farhad, C. Jie, L. Yayun, X. Allison, K. Jang-Kyo, MRS Proceedings, 2011, 1334, 2011.

[6] G. Eda, G. Fanchini, M. Chhowalla, Nat. Nanotechnol. 2008, 3, 270.

[7] R. Lv, T. Cui, M.-S. Jun, Q. Zhang, A. Cao, D. S. Su, Z. Zhang, S.-H. Yoon, J.

Miyawaki, I. Mochida, F. Kang, Adv. Funct. Mater. 2011, 21, 999.

[8] R. Jalili, S. H. Aboutalebi, D. Esrafilzadeh, K. Konstantinov, S. E. Moulton, J. M.

Razal, G. G. Wallace, ACS Nano, DOI: 10.1021/nn305906z.

[9] S. H. Aboutalebi, A. T. Chidembo, M. Salari, K. Konstantinov, D. Wexler, H. K. Liu, S. X. Dou, Energ. Environ. Sci. 2011, 4, 1855.

[10] S. H. Aboutalebi, M. M. Gudarzi, Q. B. Zheng, J.-K. Kim, Adv. Funct. Mater. 2011, 21, 2978.

[11] F. Guo, F. Kim, T. H. Han, V. B. Shenoy, J. Huang, R. H. Hurt, ACS Nano 2011, 5, 8019.

[12] Z. Xu, C. Gao, ACS Nano 2011, 5, 2908.

[13] J. E. Kim, T. H. Han, S. H. Lee, J. Y. Kim, C. W. Ahn, J. M. Yun, S. O. Kim, Angew. Chem. Int. Ed. Engl. 2011, 50, 3043.

[14] Z. Xu, C. Gao, Nat Commun. 2011, 2, 571.

[15] B. Dan, N. Behabtu, A. Martinez, J. S. Evans, D. V. Kosynkin, J. M. Tour, M.

Pasquali, I. I. Smalyukh, Soft Matter 2011, 7, 11154.

[16] G. Eda, M. Chhowalla, ACS Nano 2011, 5, 4265.

[17] Z. Xu, H. Sun, X. Zhao, C. Gao, Adv. Mater. 2013, 25, 188.

[18] L. M. Ericson, H. Fan, H. Peng, V. A. Davis, W. Zhou, J. Sulpizio, Y. Wang, R.

Booker, J. Vavro, C. Guthy, A. N. G. Parra-Vasquez, M. J. Kim, S. Ramesh, R. K. Saini, C. Kittrell, G. Lavin, H. Schmidt, W. W. Adams, W. E. Billups, M. Pasquali, W.-F. Hwang, R. H. Hauge, J. E. Fischer, R. E. Smalley, Science 2004, 305, 1447.

[19] F. Vollrath, D. P. Knight, Nature 2001, 410, 541.

[20] L. J. Cote, J. Kim, Z. Zhang, C. Sun, J. Huang, Soft Matter 2010, 6, 6096.

[21] D. van der Beek, H. N. W. Lekkerkerker, Langmuir 2004, 20, 8582.

[22] F. M. van der Kooij, H. N. W. Lekkerkerker, J. Phys. Chem. B 1998, 102, 7829.

[23] S. Paasch, F. Schambil, M. J. Schwuger, Langmuir 1989, 5, 1344.

[24] R. Mezzenga, C. Meyer, C. Servais, A. I. Romoscanu, L. Sagalowicz, R. C. Hayward, Langmuir 2005, 21, 3322.

[25] R. Jalili, J. M. Razal, P. C. Innis, G. G. Wallace, Adv. Funct. Mater. 2011, 21, 3363.

[26] M. D. Kempe, N. R. Scruggs, R. Verduzco, J. Lal, J. A. Kornfield, Nat. Mater. 2004, 3, 177.

[27] J. M. Razal, K. J. Gilmore, G. G. Wallace, Adv. Funct. Mater. 2008, 18, 61.

[28] R. Jalili, J. M. Razal, G. G. Wallace, J. Mater. Chem. 2012, 22, 25174.

[29] D. Esrafilzadeh, J. M. Razal, S. E. Moulton, E. M. Stewart, G. G. Wallace, J. Control. Release, DOI: 10.1016/j.jconrel.2013.01.022.

[30] V. A. Davis, A. N. G. Parra-Vasquez, M. J. Green, P. K. Rai, N. Behabtu, V. Prieto, R. D. Booker, J. Schmidt, E. Kesselman, W. Zhou, H. Fan, W. W. Adams, R. H. Hauge, J. E. Fischer, Y. Cohen, Y. Talmon, R. E. Smalley, M. Pasquali, Nat. Nanotechnol. 2009, 4, 830. [31] S. Park, K.-S. Lee, G. Bozoklu, W. Cai, S. T. Nguyen, R. S. Ruoff, ACS Nano 2008, 2, 572.

[32] J. P. Rourke, P. A. Pandey, J. J. Moore, M. Bates, I. A. Kinloch, R. J. Young, N. R. Wilson, Angew. Chem. Int. Ed. Engl. 2011, 50, 3173.

[33] S. H. Aboutalebi, S. Aminorroaya-Yamini, I. Nevirkovets, K. Konstantinov, H. K. Liu, Adv. Energy Mater. 2012, 2, 1439. 
[34] X. Fan, W. Peng, Y. Li, X. Li, S. Wang, G. Zhang, F. Zhang, Adv. Mater. 2008, 20, 4490.

[35] X. Lin, X. Shen, Q. Zheng, N. Yousefi, L. Ye, Y.-W. Mai, J.-K. Kim, ACS Nano 2012, 6, 10708.

[36] D. A. Dikin, S. Stankovich, E. J. Zimney, R. D. Piner, G. H. B. Dommett, G.

Evmenenko, S. T. Nguyen, R. S. Ruoff, Nature 2007, 448, 457.

[37] M. K. Shin, B. Lee, S. H. Kim, J. A. Lee, G. M. Spinks, S. Gambhir, G. G. Wallace, M.

E. Kozlov, R. H. Baughman, S. J. Kim, Nat. Commun. 2012, 3, 650.

[38] C. Xiang, N. Behabtu, Y. Liu, H. G. Chae, C. C. Young, B. Genorio, D. E.

Tsentalovich, C. Zhang, D. V. Kosynkin, J. R. Lomeda, C.-C. Hwang, S. Kumar, M. Pasquali, J. M. Tour, ACS Nano 2013, 7, 1628.

[39] Z. Dong, C. Jiang, H. Cheng, Y. Zhao, G. Shi, L. Jiang, L. Qu, Adv. Mater. 2012, 24, 1856.

[40] M. Zhang, K. R. Atkinson, R. H. Baughman, Science 2004, 306, 1358.

[41] J. Hone, M. C. Llaguno, N. M. Nemes, A. T. Johnson, J. E. Fischer, D. A. Walters, M. J. Casavant, J. Schmidt, R. E. Smalley, Appl. Phys. Lett. 2000, 77, 666.

[42] Q. Liang, X. Yao, W. Wang, Y. Liu, C. P. Wong, ACS Nano 2011, 5, 2392.

[43] A. A. Balandin, S. Ghosh, W. Bao, I. Calizo, D. Teweldebrhan, F. Miao, C. N. Lau, Nano Lett. 2008, 8, 902.

[44] A. A. Balandin, Nat. Mater. 2011, 10, 569.

[45] Z. Wei, Z. Ni, K. Bi, M. Chen, Y. Chen, Carbon 2011, 49, 2653.

[46] S. Iijima, Nature 1991, 354, 56.

[47] N. Miyamoto, T. Nakato, J. Phys. Chem. B 2004, 108, 6152. 

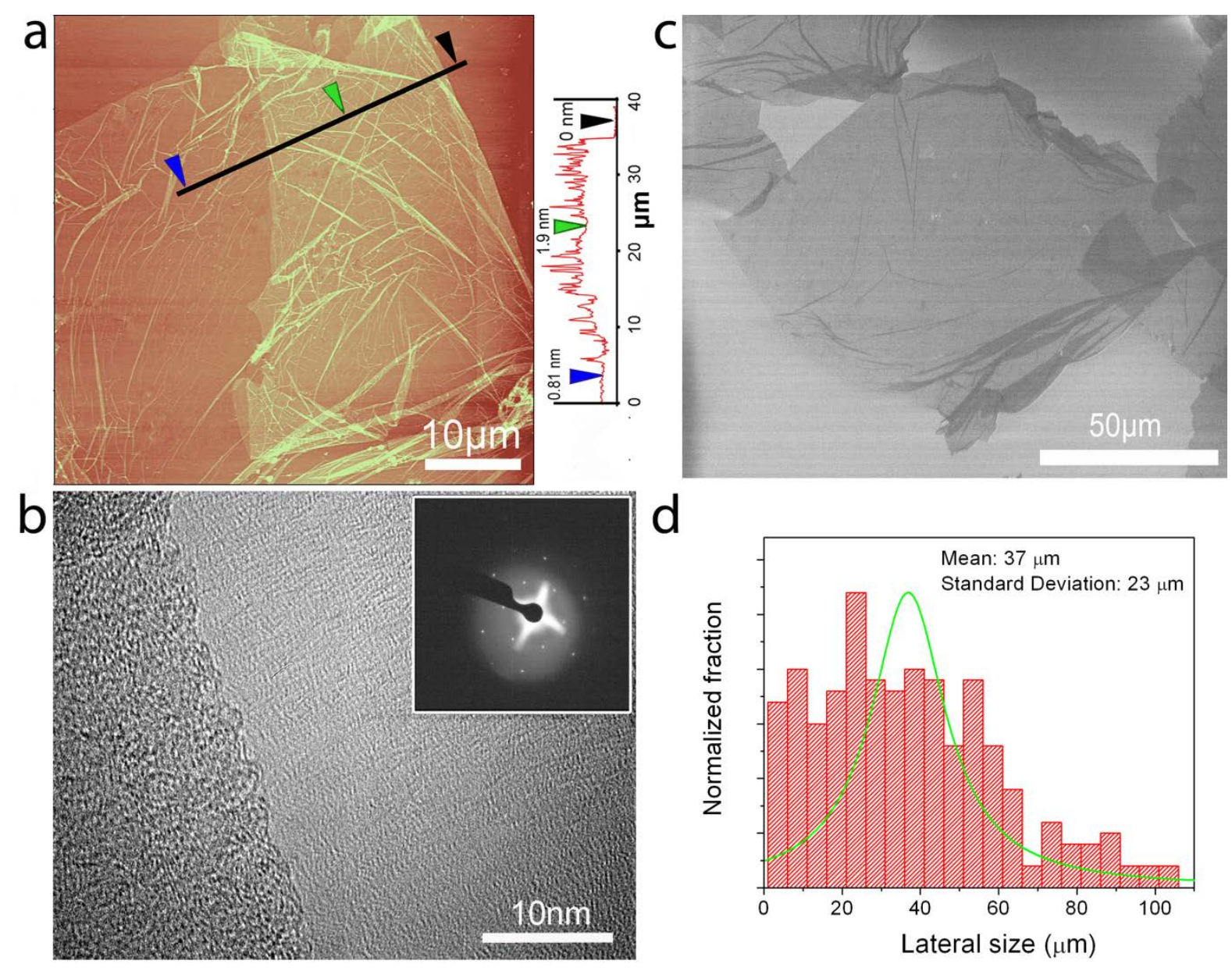

Figure 1. As-prepared GO dispersions predominantly contain giant monolayer GO sheets. a) AFM image of a large GO sheet with lateral size $>75 \mu \mathrm{m}$. Inset shows the height profile derived from the marked line in the AFM image showing monolayer GO sheet thickness of 0.81 nm. b) High-resolution TEM image of monolayer GO sheet and the corresponding electron diffraction pattern (inset). c) Representative SEM images of GO sheets present in asprepared GO dispersions contain GO sheets with lateral size as large as $100 \mu \mathrm{m}$, d) The corresponding lateral size distribution of GO sheets (the diameter of an equal-area circle). 


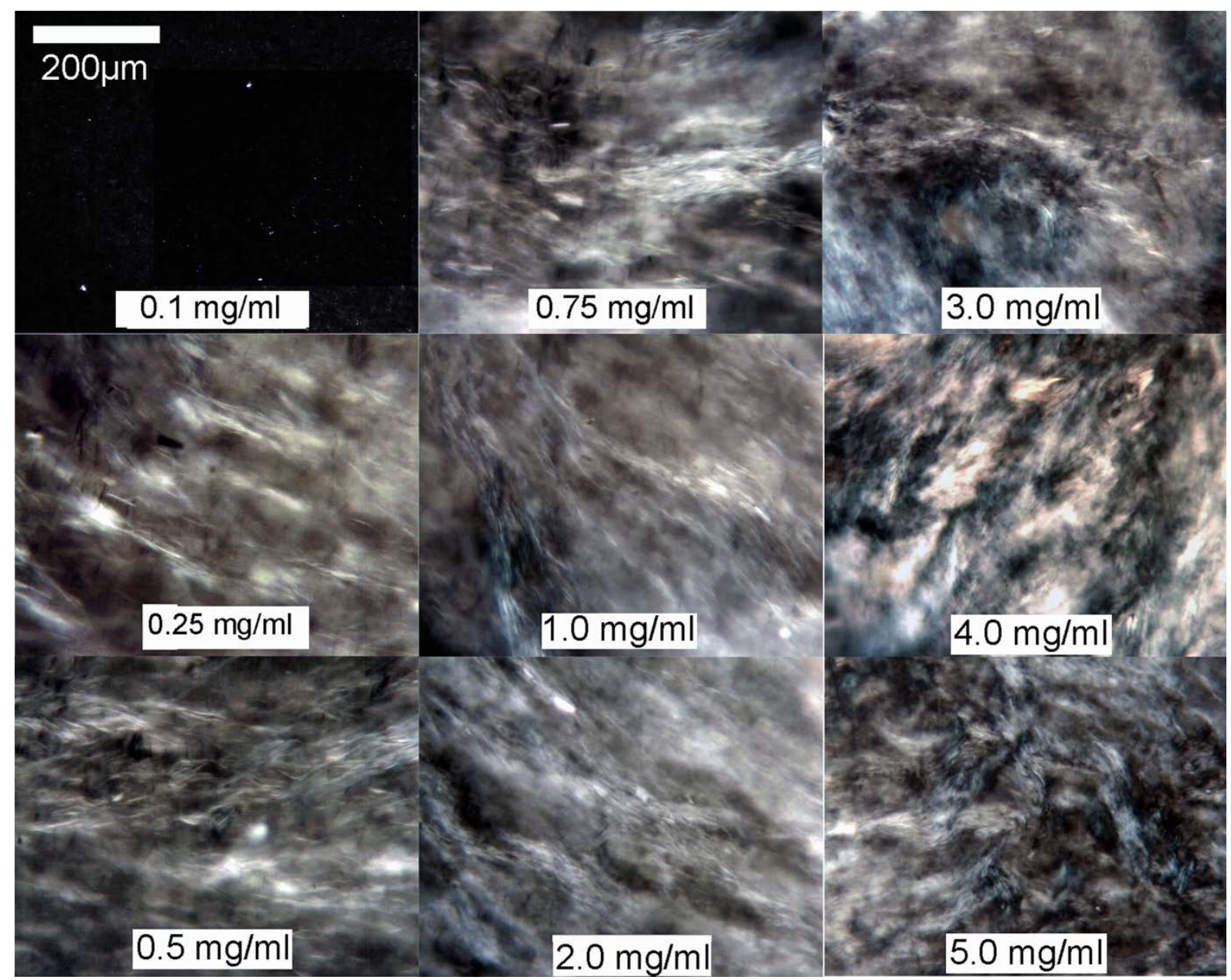

Figure 2. Representative polarized optical micrographs of aqueous liquid crystalline graphene oxide (GO) dispersions at various GO concentrations (0.1 to $5.0 \mathrm{mg} \mathrm{ml}^{-1}$ ) evidenced LC formation at concentration as low as $0.25 \mathrm{mg} \mathrm{ml}^{-1}$. 
a

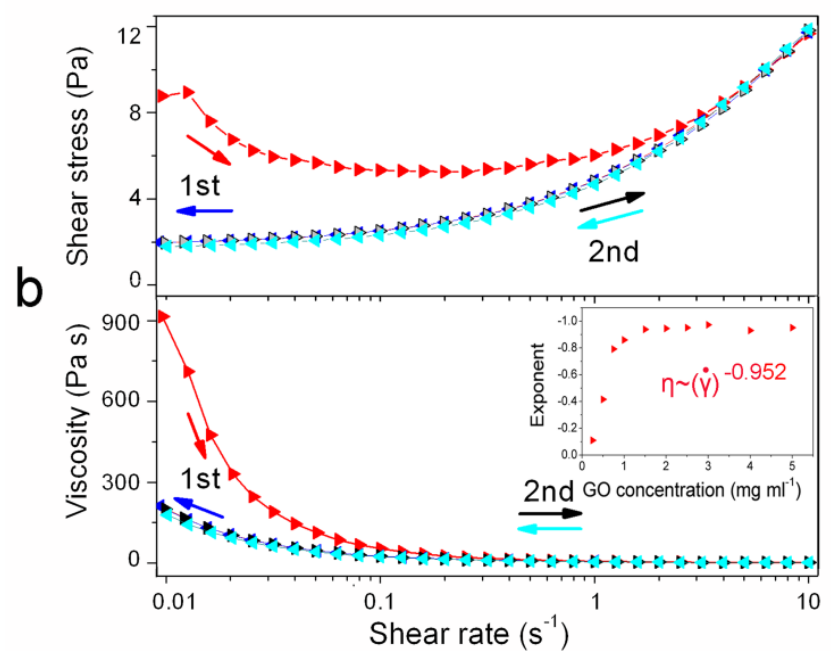

C $\quad \mathrm{GO}$ concentration $\left(\mathrm{mg} \mathrm{ml}^{-1}\right)$
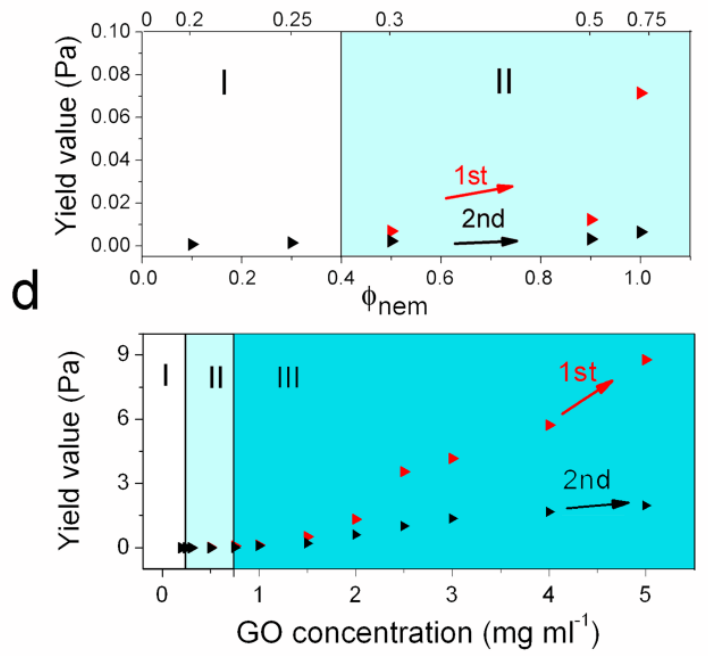

Figure 3. a) Shear stress and b) Viscosity vs. shear rate of $5 \mathrm{mg} \mathrm{ml}^{-1}$ aqueous LC GO dispersions showing the first two complete ascending-descending flow curves in logarithmic scale (arrows represent direction of flow curve measurement). The inset in b is the exponent part of the fit of viscosity as a function shear rate in different concentration $\left(\eta \sim(\dot{\gamma})^{-0.952}\right.$ is for $5 \mathrm{mg} \mathrm{ml}^{-1}$ ). c and d) Yield value of aqueous LC GO dispersions for the first and second ascending flow curves. Regions I, II, and III correspond to non-spinnable, slightly spinnable, and fully spinnable GO dispersions. c) Yield value as a function of nematic phase volume fraction, $\varphi_{\text {nem }}$ (main x-axis) and the corresponding GO concentration (x-axis shown on top) which fall within the identified regions I (un-spinnable) and II (partially spinnable), d) Yield value at various GO concentrations. Red and black triangles refer to the data obtained from the first and second ascending flow curves, respectively. 

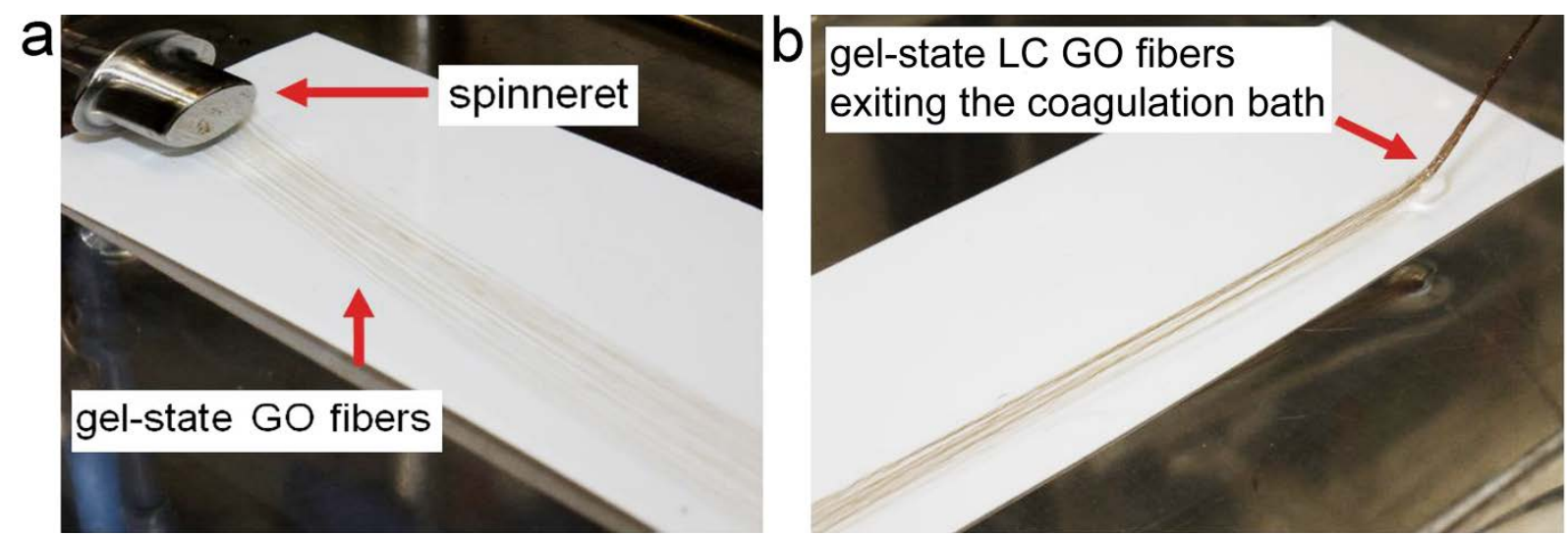

Figure 4. Digital images of GO yarns produced using a multi-hole spinneret. a) Snapshot of the formation of gel-state GO filaments as they exit the multi-hole spinneret and b) When they are being pulled out from the bath for collection. 

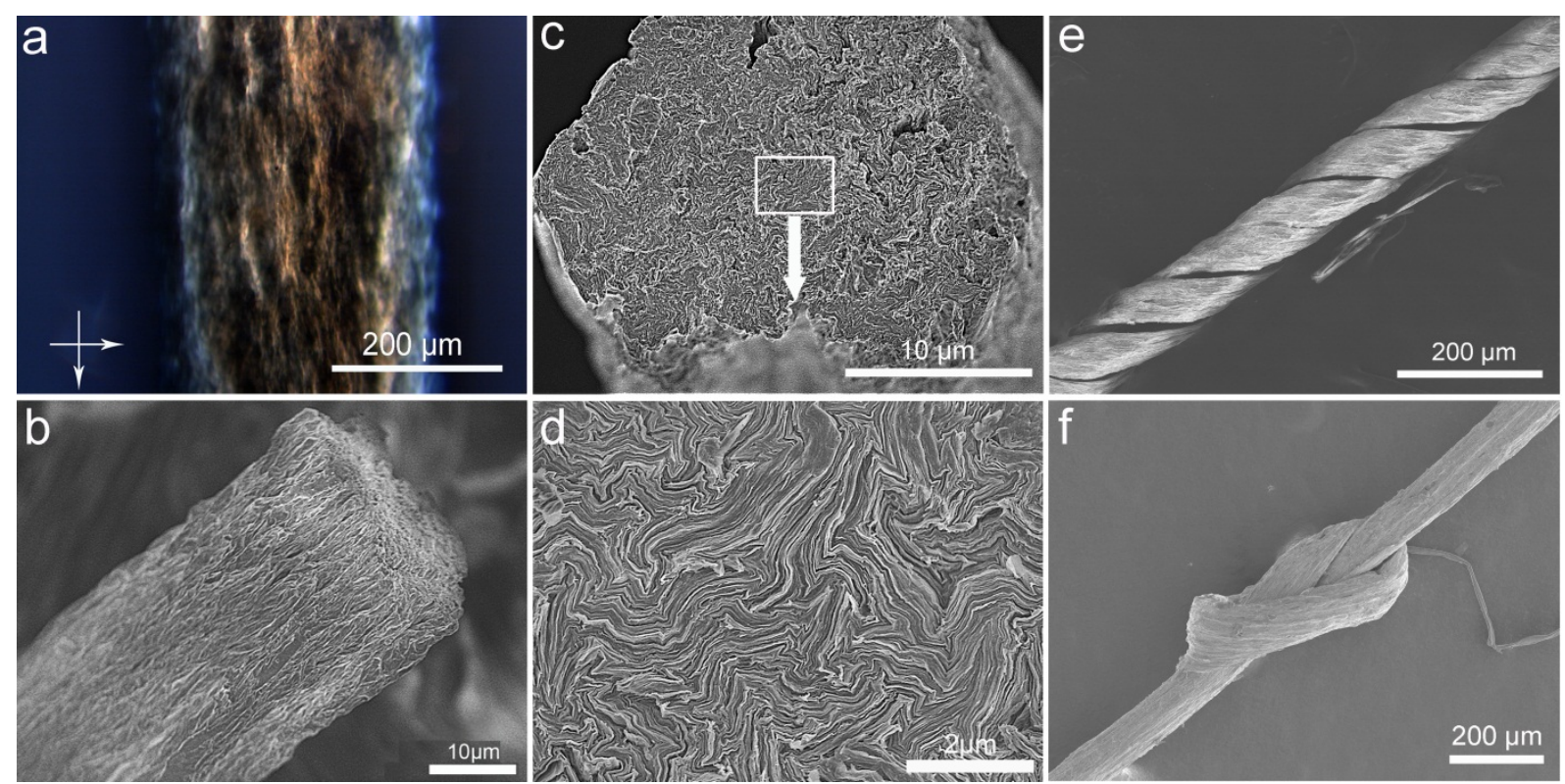

Figure 5. a) Polarized optical micrograph of as-spun gel-state GO fiber showing birefringence.

Birefringence properties confirmed ordered LC domains were formed and preserved during spinning process (arrows show polarizers direction). SEM images of an as-spun GO fiber showing b) Corrugated surface and c) Near-circular cross-section. d) Close-up SEM image of the cross-section of GO fiber shown in b revealing GO sheet planes that are oriented along the fiber axis. e-f) SEM images of crumpled and knotted rGO yarns (reduced by annealing) showing their flexibility. 


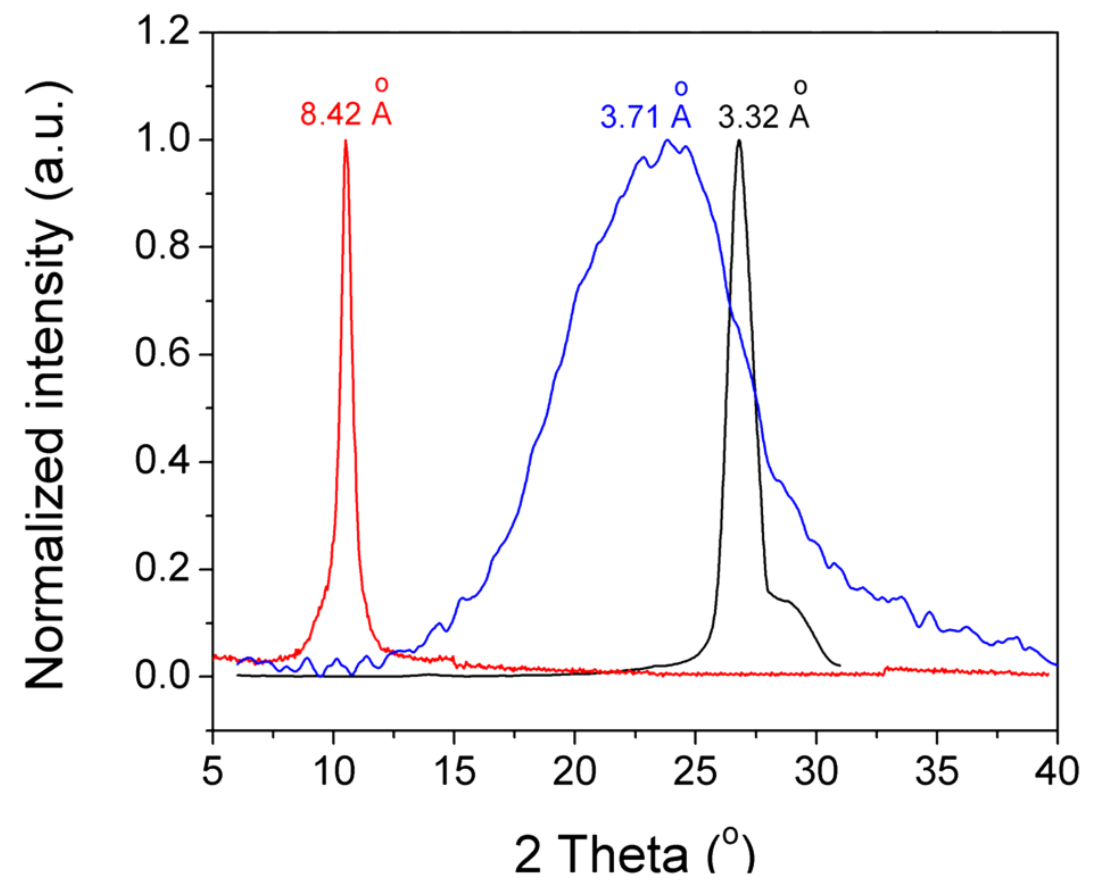

Figure 6. Comparison of XRD patterns of GO fiber (8.42 $\AA$ ), rGO fiber (3.71 $\AA$ ), and thermally-expanded graphite (3.32 ^). 

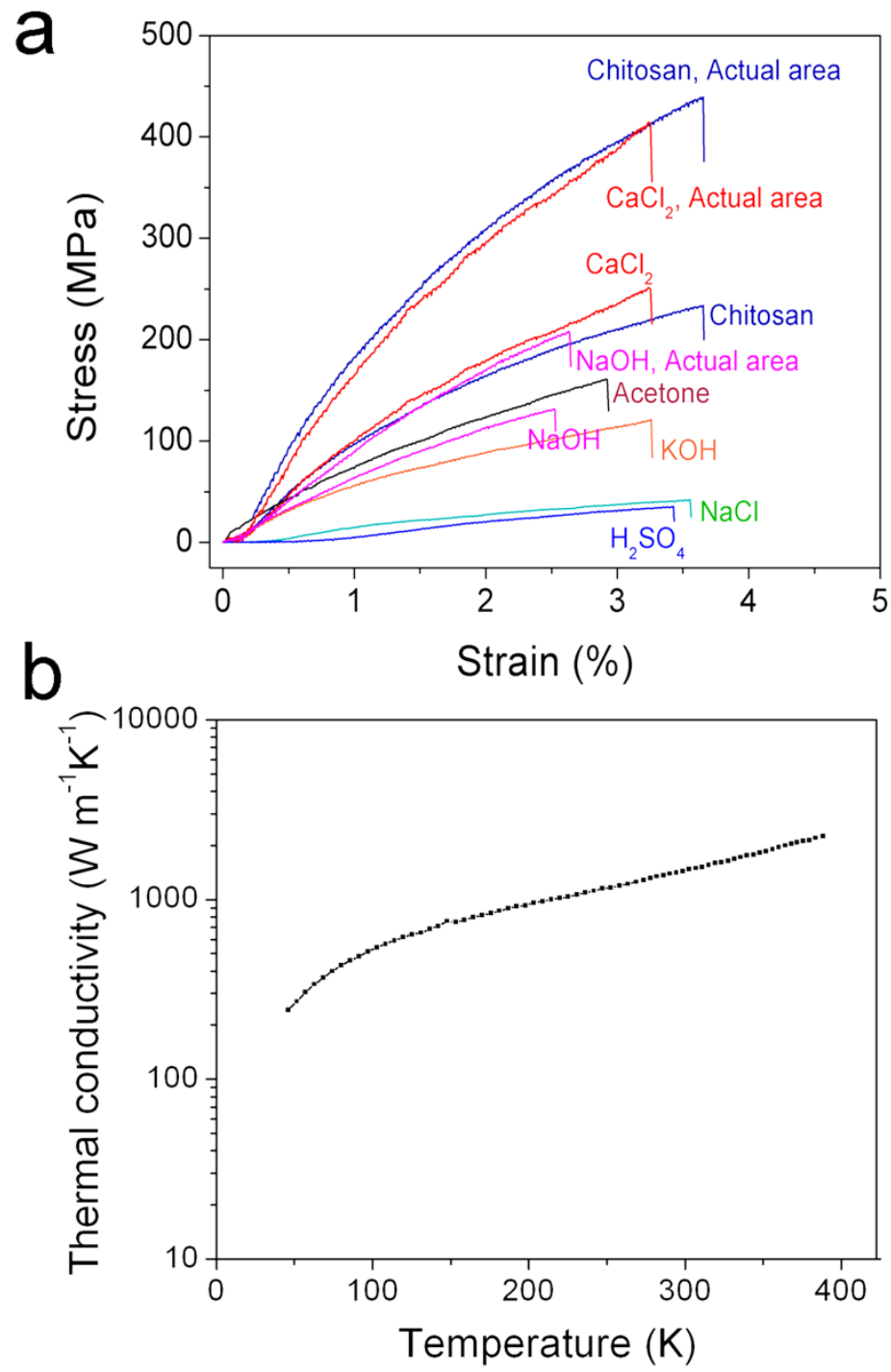

Figure 7. Properties of GO and rGO fibers. a) Stress-strain curves of as-spun GO fibers prepared using various coagulation baths b) Temperature-dependent thermal conductivity of rGO fiber in the 46 to $380 \mathrm{~K}$ range. 
Table 1. Mechanical, electrical and thermal properties of GO and rGO fibers prepared in this study and previous reports.

\begin{tabular}{|c|c|c|c|c|c|c|}
\hline Production condition & $\begin{array}{l}\text { Young's } \\
\text { Modulus }\end{array}$ & $\begin{array}{c}\text { Ultimate } \\
\text { Stress } \\
\end{array}$ & Toughness & $\begin{array}{c}\text { Electrical } \\
\text { conductivity }\end{array}$ & $\begin{array}{l}\text { Thermal } \\
\text { conductivity }\end{array}$ & Reference \\
\hline & {$[\mathrm{GPa}]$} & {$[\mathrm{MPa}]$} & {$\left[\mathrm{J} \mathrm{g}^{-1}\right]$} & {$\left[\mathrm{S} \mathrm{m}^{-1}\right]$} & {$\left[\mathrm{W} \mathrm{m} \mathrm{m}^{-1} \mathrm{~K}^{-1}\right]$} & \\
\hline GO fibers coagulated by Chitosan & $22.6 \pm 1.9$ & $442 \pm 18$ & 4.8 & - & - & This study \\
\hline GO fibers coagulated by $\mathrm{CaCl}_{2}$ & $20.1 \pm 2.1$ & $412 \pm 30$ & 4.8 & - & - & This study \\
\hline rGO fibers coagulated by $\mathrm{NaOH}$ & $11 \pm 2.4$ & $183 \pm 25$ & 1.6 & 221 & - & This study \\
\hline $\begin{array}{l}\text { rGO fibers coagulated by } \mathrm{NaOH} \text { (further reduction } \\
\text { at } 220^{\circ} \mathrm{C} \text { ) }\end{array}$ & $9.0 \pm 2.1$ & $115 \pm 19$ & 1.3 & $2.8 \times 10^{2}$ & 1435 & This study \\
\hline Graphene nanoribbons fibers annealed at $1500^{\circ} \mathrm{C}$ & 36.2 & 378 & - & $2.8 \times 10^{3}$ & - & [38] \\
\hline $\begin{array}{l}\text { rGO fiber (glass pipeline moulding, annealed at } \\
\qquad 230^{\circ} \mathrm{C} \text { ) }\end{array}$ & - & 180 & - & $10^{3}$ & - & [39] \\
\hline $\begin{array}{l}\text { rGO fiber (glass pipeline moulding, annealed at } \\
\qquad 800^{\circ} \mathrm{C} \text { ) }\end{array}$ & - & 420 & - & - & - & [39] \\
\hline rGO fiber (reduced by hydroiodic acid) & 5.4 & 130 & - & $2.5 \times 10^{4}$ & - & [14] \\
\hline GO fibers coagulated by $\mathrm{CaCl}_{2}$ (stretched) & 6.3 & 364 & - & - & - & {$[17]$} \\
\hline $\begin{array}{l}\text { rGO fibers coagulated by } \mathrm{CaCl}_{2} \text { (reduced by } \\
\text { hydroiodic acid and stretched) }\end{array}$ & 11.2 & 501 & - & $4.1 \times 10^{4}$ & - & [17] \\
\hline
\end{tabular}




\section{The table of contents entry}

We provide new insights in the processing of liquid crystalline graphene oxide (GO) dispersion (containing large GO sheets) demonstrating a facile and scalable production of GO and reduced GO fibers and yarns with exciting properties such as high thermal conductivity. These results provide a universal platform for the development of solution-based processing methods, properties and applications of LC GO-based architectures.

Keywords: graphene oxide, wet-spinning, fiber, liquid crystal

Rouhollah Jalili, Seyed Hamed Aboutalebi, Dorna Esrafilzadeh, Roderick L. Shepherd, Jun Chen, Sima Aminorroaya-Yamini, Konstantin Konstantinov, Andrew I. Minett, Joselito M. Razal* and Gordon G. Wallace *

Scalable One-Step Wet-spinning of graphene Fibers and Yarns from Liquid Crystalline

Dispersions of Graphene Oxide: Towards Multifunctional Textiles

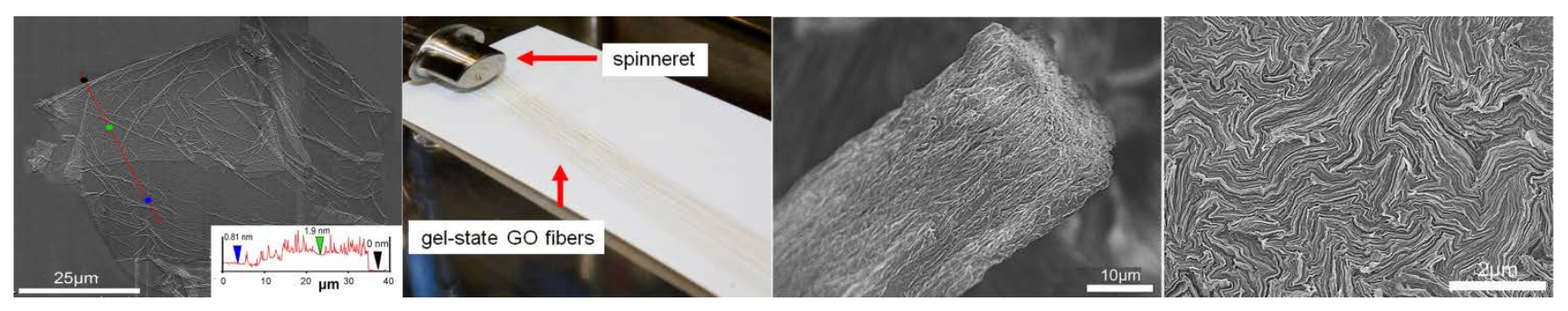

